\title{
Terapias hormonales utilizadas en el control artificial de la madurez sexual en peces de cultivo: una revisión
}

\author{
Hormone therapy for the artificial control of sexual maturity in fish culture: a review
}

\author{
I Valdebenito* \\ Escuela de Acuicultura, Universidad Católica de Temuco, Temuco, Chile. \\ Centro de Genómica Nutricional Agroacuícola, CGNA.
}

\begin{abstract}
SUMMARY
The present literature review analyzes the current state of artificial control of sexual maturity in fish culture using hormone therapy, evaluating the advantages and disadvantages of different technological strategies available. The discussion covers from the first successful attempt conducted by Houssay (1930), Argentina, up to the most recent and more effective techniques of slow release and the use of dopamine inhibitors. In addition, the evaluation of the use of non traumatic methodologies that up until now have not been entirely effective is covered.
\end{abstract}

Palabras clave: reproducción, peces, GnRH, sincronización, desove.

Key words: fish reproduction, GnRH, spawning, induction.

\section{INTRODUCCION}

El perfil de desarrollo que cada día adquiere la acuicultura industrial en Chile y en el mundo, con un alto desarrollo tecnológico y un amplio conocimiento de las especies que se cultivan, exige como requisito un adecuado control de los procesos reproductivos de los peces (Zohar y Mylonas 2001). Ya a inicios de la década de los 80, Harvey y Hoar (1980) planteaban que una actividad acuícola comercial no se podía sostener en la captura de larvas o juveniles desde el medio natural. Una vez controlada la reproducción de una especie acuática en sistemas de cultivo, se pueden producir los descendientes que permitirán iniciar ciclos nuevos y permanentes de producción. Asociado a ello, se podrán aplicar programas de selección de razas de mejor crecimiento, modificar su época de reproducción (Davy y Chouinard 1980, Billard y Breton 1985, Bromage 1995) o aplicar programas de manipulación genética que permitan mejorar la sobrevivencia, la calidad de su carne (Thorgaard 1995), reducir los problemas de la maduración sexual, generar poblaciones monosexo y, últimamente, producir organismos genéticamente modificados (Donaldson y col 1996, Donaldson 1997, Solar 2002).

Desgraciadamente, muchas especies mantenidas en sistemas de cultivo artificial sufren disfunciones reproductivas importantes. Más comúnmente, las hembras inician el desarrollo gonadal en forma normal, pero no alcanzan la maduración final del oocito, la ovulación y/o

Aceptado: 23.01.2008.

* ivisler@uctemuco.cl la puesta (Zohar 1988, Peter y col 1993). Los machos, por su parte, aunque mucho más resistentes a las condiciones ambientales del cultivo y al estrés producido por éste, producen un menor volumen de semen, o bien éste es de mala calidad (Billard 1986, 1989). Manipulaciones de algunos factores ambientales como el fotoperiodo, temperatura, salinidad, volumen del tanque, vegetación del sustrato, etc., pueden a menudo mejorar la calidad de las puestas (Donaldson y Hunter 1983, Zanuy y col 1995, Donaldson y col 1996, Donaldson 1997, Prat y col 1999, Rodríguez y col 2000, Bromage y col 2001, Mylonas y Zohar 2001 ab, Zohar y Mylonas 2001). Sin embargo, en algunas especies los tratamientos hormonales son la única alternativa de lograr adecuados procesos reproductivos en los peces que se cultivan. En los últimos años, una variedad de hormonas y tecnologías aplicadas a su uso han sido utilizadas exitosamente en numerosas especies (Hoar 1969, Donaldson y Hunter 1983, Mylonas y Zohar 2001 $1^{\text {ab }}$, Zohar y Mylonas 2001). Estos métodos comienzan en 1930 con la inyección de extracto crudo de hipófisis de peces maduros (con altos niveles de GtH) con el fin de inducir la puesta (Zohar y Mylonas 2001). Hoy son utilizados varios compuestos sintéticos de alta potencia de la hormona liberadora de gonadotrofina $(\mathrm{GnRHa})$ que aplicados desde simples inyecciones en suero fisiológico hasta en microesferas han solucionado numerosos problemas reproductivos en varias especies (Zohar 1988, Van Winkoop y col 1994, Donaldson y col 1996, Montero y Dufour 1996, Estay y col 1996, Donaldson 1997, Kitahashi y col 1998, Yambe y col 1999, Forniés y col 2001, Patiño y col 2001, Solar 2002, entre otros). 


\section{CONTROL ENDOCRINO DE LA REPRODUCCION EN PECES}

En peces, como en todos los vertebrados, la reproducción es regulada por el cerebro a través de la hormona liberadora de gonadotrofina $(\mathrm{GnRH})$ que se produce específicamente en el hipotálamo (Harvey y Hoar 1980, Zohar 1988, Montero y Dufour 1996, Donaldson 1997, Peter y Yu 1997). Este decapéptido estimula la producción de gonadotrofinas $(\mathrm{GtH})$ por parte de la hipófisis o pituitaria. En algunos peces la dopamina es un inhibidor negativo de la producción y liberación de GtH por parte de la hipófisis (Zohar 1988, Redding y Patiño 1993, Peter y Yu 1997). El control de la reproducción que realiza esta glándula es a través de dos hormonas gonadotróficas: la hormona folículo estimulante (FSH o $\mathrm{GtH}$ I), que regula la vitelogénesis en hembras y la espermatogénesis en los machos, y la hormona luteinizante (LH o GtH II), encargada de controlar la maduración final del oocito en las hembras (Peter y Yu 1997).

\section{HEMBRAS}

Durante la vitelogénesis, la FSH o LH estimulan la producción de testosterona ( $\mathrm{T}$ ) en las células de la teca y su aromatización a $17 \beta$-estradiol (E2) en las células de la granulosa del folículo ovárico. El incremento de E2 en el plasma estimula la producción de vitelogenina (VGT) en el hígado, la que es reconocida por receptores de membrana e incorporada por el oocito a través de micropinocitosis, proceso que es regulado por la FSH. Al finalizar la vitelogénesis, incrementan los niveles plasmáticos de LH que produce un marcado aumento de los niveles plasmáticos del esteroide inductor de la maduración (MIS) que actúa a nivel de la membrana del oocito induciendo su maduración final (FOM) (Gordon y col 1987, Peter y Yu 1997,

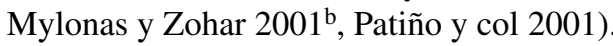

\section{MACHOS}

Las gonadotrofinas regulan la espermatogénesis a través de la producción de andrógenos por el testículo, específicamente de la 11-ketotestosterona (11-KT) por las células de Leydig que se encuentran en la periferia de los lóbulos testiculares. La T es un precursor de la 11-KT y sus niveles plasmáticos varían durante el ciclo reproductivo en las diferentes especies, encontrándose una tendencia general de un marcado aumento de los niveles plasmáticos de 11-KT durante la espermatogénesis y una disminución antes o durante el periodo de espermiación. La acción de la 11-KT llega finalmente a estimular las células de Sertoli que son las encargadas de activar la espermatogénesis (Mylonas y Zohar 2001 b). En forma similar a lo ocurrido en hembras, un incremento en los niveles plasmáticos de LH al comienzo de la estación de desoves causa un aumento en la esteroidogénesis a nivel testicular junto con la producción de MIS (Nagahama 1994). La LH y el MIS inducen un incremento en los niveles de semen producidos a través de la estimulación de la producción del plasma seminal, y el MIS estimula la capacidad de movimiento del espermatozoide a través de un ascenso del pH en el plasma seminal (Miura y col 1995).

\section{ALTERACIONES EN EL PROCESO REPRODUCTIVO DE PECES DE CULTIVO}

Regularmente, las condiciones ambientales inadecuadas, manejo, alimentación y el estrés del cultivo generan dificultades al proceso normal de reproducción de los organismos mantenidos en cautiverio, los que afectan en forma más marcada a las hembras que a los machos (Estay 1988), produciendo alteraciones que según Zohar y Mylonas (2001) pueden alcanzar los siguientes niveles:

- Ausencia de desarrollo gonadal, como lo ocurrido en la anguila europea (Anguilla sp.) que en condiciones de cultivo no alcanza a desarrollar la vitelogénesis ni espermatogénesis (Fontaine 1975, Zohar y Mylonas 2001). De forma similar, la Seriola del Mediterráneo (Seriola dumerili) es una especie de gran interés para la acuicultura (Abellan y Basurco 1999) y los especímenes capturados en la naturaleza y después de unos pocos años mantenidos en condiciones de cultivo parecen iniciar el proceso de vitelogénesis, pero sólo un pequeño porcentaje de hembras desarrolla oocitos que sobrepasen los 200-300 $\mu \mathrm{m}$ de diámetro, los que ingresan en un proceso de atresia y son absorbidos al final de la temporada de puesta (Zohar y Mylonas 2001).

- Ausencia de la maduración final de los oocitos. Este es uno de los problemas más frecuentes observados en las especies de interés comercial para la acuicultura. Las hembras parecen iniciar normalmente el proceso de vitelogénesis, pero al comienzo de la estación de puesta los oocitos postvitelogénicos detienen su maduración final y se transforman en atrésicos. Estos tipos de alteraciones han sido observados en algunas especies de serránidos por Tucker (1994), en el lenguado de verano Paralichthys lethostigma (Berlinsky y col 1997), en el lenguado de cola amarilla Pleuronectes ferrugineus (Larsson y col 1997), entre otras especies.

- Ausencia de desove. Las especies que presentan este problema pueden desarrollar normalmente la vitelogénesis, la maduración final de los oocitos y la ovulación, pero no son capaces de realizar la puesta (Gordon y col 1987, Zohar y Mylonas 2001). Debido a lo anterior, los oocitos maduros deben ser extraídos manualmente desde la cavidad abdominal de la hembra (Edwards 1978, Gordon 1987, Estay y col 1994 y Estay y col $\left.1995^{\mathrm{ab}}\right)$. Esta dificultad se presenta en el rodaballo Scophthalmus maximus (Kjorsvik y col 1990). La viabilidad de los oocitos mientras se encuentran en la cavidad abdominal es dependiente de la temperatura 
y muy variable entre una especie y otra. Por ejemplo, en salmónidos alcanza varios días (Gordon y col 1987) y en otras, sólo de algunas horas, como en tilapias y lenguados (Bromage 1995).

En la mayoría de las especies los machos se adaptan mejor que las hembras a las condiciones de cultivo a las que son sometidos y son capaces de madurar y producir gametos viables con mayor facilidad. Estay (1988) determina en una población de reproductores de trucha arcoiris cultivada en agua con temperatura constante de $16^{\circ} \mathrm{C}$, que las hembras no son capaces de producir gametos viables, en cambio los machos sí producen semen de buena calidad.

Con la excepción de la anguila europea, las alteraciones reproductivas de los machos se centran en una reducción de la cantidad y calidad de los gametos producidos (Zohar y Mylonas 2001). Por ejemplo, los machos de la lubina americana mantenidos en cautiverio exhiben una reducción significativa en la cantidad de semen producido (Mylonas y col $\left.1998^{\mathrm{ab}}\right)$. También el lenguado de cola amarilla ( $P$. ferrugineus) y rodaballo producen menos de $1,0 \mathrm{ml}$ de semen por pez (Suquet y col 1992, Brown y Crim 1998). Algunas variedades de salmón del Atlántico seleccionadas genéticamente por su rápido crecimiento producen menos de 0,1 ml de semen por kilogramo de peso corporal (Zohar 1996). Especialmente los reproductores capturados de la naturaleza producen semen con espermatozoides inmóviles (Berlinsky y col 1997), o bien un semen de alta viscosidad que es difícil de mezclar con el medio acuoso en el que se realiza la fertilización (Vermeirssen y col 1998, Vermeirssen y col 2000).

El estrés del cautiverio en los peces puede afectar el sistema inmune, el crecimiento y la reproducción, alterando varios niveles del sistema endocrino, o el desarrollo de gametos y su calidad, alterando la sobrevivencia de huevos y larvas y su desarrollo (Foo y Lam 1993, Campbell y col 1994, Pankhurst y Van der Kraak 1997, Schreck y col 2001, Wagner y col 2002). Los autores anteriores reconocen a los niveles plasmáticos de cortisol como un indicador del nivel de estrés de los peces; según esto, Schreck y col (2001), en base a una amplia revisión bibliográfica, informan que en trucha arcoiris los niveles de cortisol después de un agente estresante son mayores en peces que se encuentran cerca del proceso de maduración sexual y, además, mucho más marcado en hembras que en machos.

\section{TECNICAS HORMONALES UTILIZADAS EN EL CONTROL DE LA REPRODUCCION EN PECES DE CULTIVO}

El uso de hormonas para el control de la reproducción en peces se ha centrado en la inducción de la maduración final del oocito (FOM), de la ovulación, la espermiación y la puesta en peces que no son capaces de completar su ciclo reproductivo en agua dulce, o bien como una forma de optimizar el manejo (rendimiento) productivo de una piscicultura, adelantando el proceso de maduración, ovulación y desove en algunas semanas. Por ejemplo, los salmónidos son peces que pueden ovular en cautiverio, pero no desovar. Por lo tanto, este último proceso debe ser realizado manualmente durante los meses que se prolonga el período de puesta. En estos peces, la ovulación es inducida artificialmente con el fin de reducir el manejo, el estrés de los peces y las altas mortalidades producidas en este período de alta manipulación. Además de esto, permite reducir la duración del período de puestas y el nivel de trabajo del personal de la piscicultura (Donaldson 1997). Otra aplicación de la manipulación hormonal es la colección de gametos para la hibridación interespecífica a través de fertilización artificial con especies que usualmente no desovan en sistemas de cultivo, o bien en programas de selección genética que requieren de fertilización artificial dirigida para dos grupos de peces diferentes. De esta manera, la manipulación hormonal de la reproducción en peces es específica para la inducción de la ovulación, espermiación y puesta, y continuarán jugando un importante papel en el manejo comercial de reproductores en sistemas de cultivo (Donaldson y col 1996, Donaldson 1997, Zohar y Mylonas 2001).

Preparados de gonadotrofinas $(G t H)$. Los primeros estudios sobre la posibilidad de usar hormonas exógenas para inducir la maduración final del oocito (FOM) y la ovulación en peces fueron realizados por Houssay en 1930 (Harvey y Hoar 1980, Zohar y Mylonas 2001), quien inyectó hembras con hipófisis de otras especies y observó que ovulaban anticipadamente. Este trabajo es el comienzo de una serie de métodos utilizados para la inducción a la puesta en peces, comenzando con la técnica de hipofisación.

Hipofisación y extractos de hipófisis de peces. La hipofisación, el uso de extractos de hipófisis para inducir la puesta en peces, comienza a fines de la década de 1930 en Brasil, en la década siguiente en USA y a inicios de 1950 en Japón (Zohar y Mylonas 2001). La recolección de hipófisis se realiza desde peces sexualmente maduros, tanto machos como hembras, encontrándose que las glándulas colectadas durante el periodo de puesta son más efectivas en inducir la ovulación, lo que demuestra la acumulación de $\mathrm{GtH}$ (principalmente $\mathrm{LH}$ ) en la hipófisis antes y durante la estación de desoves. Las glándulas colectadas deben ser almacenadas en alcohol o deshidratadas en acetona. Al momento de ser inyectadas en el pez, deben ser disueltas en solución fisiológica (Davy y Chouinard 1980, Pagán-Font y Zimet 1985, Courtois y col 1986) y aplicadas al pez mediante una inyección intramuscular o intraperitoneal (Harvey y Hoar 1980, Amutio y col 1986). Las dosis utilizadas en principio fueron de una glándula por pez macho y 1,5 glándulas por pez hembra. Sin embargo, estas dosis no siempre han sido efectivas debido a las variaciones en el tamaño de los peces receptores y la concentración de GtH existente en las glándulas a inyectar 
(Patiño 1997). Regularmente, las cantidades a suministrar se aplican en dos o cuatro dosis inyectadas entre algunas horas o días (Davy y Chouinard 1980, Pagán-Font y Zimet 1985, Amutio y col 1986).

En la actualidad esta metodología es utilizada masivamente en la producción de carpas en países asiáticos y el método de hipofisación ha sido estandarizado a dos inyecciones: primero, una inyección pequeña de un 10 a $20 \%$ y segundo, una mayor aplicada 12 a 24 hrs después. Las dosis más efectivas utilizadas se encuentran entre 2 a $10 \mathrm{mg}$ de pituitaria por cada $\mathrm{kg}$ de pez receptor (Sower y Schreck 1980, Courtois y col 1986, Zohar y Mylonas 2001).

No obstante, esta técnica está asociada con varias desventajas, dentro de las cuales Carrillo y Zanuy (1995) y Zohar y Mylonas (2001) señalan como las más importantes:

- Una gran variación en el contenido de LH de la pituitaria.

- La administración de hormonas adicionales presentes en la pituitaria que pueden afectar adversamente la fisiología de los peces tratados.

- La potencial transmisión de enfermedades desde el pez donante al receptor.

\section{GONADOTROFINAS EXOGENAS}

Los problemas señalados anteriormente con las técnicas de hipofisación llevaron a desarrollar la obtención de preparados de LH, total o parcialmente purificados, a través de separación cromatográfica, aun cuando incorpora un alto costo al producto final. Preparaciones purificadas de gonadotrofinas de salmón y carpa han sido comercializadas hace algún tiempo (Harvey y Hoar 1980, Davy y Chouinard 1980, Peter y Yu 1997, Zohar y Mylonas 2001). Sin embargo, debido a la alta especificidad de las gonadotrofinas se han obtenido escasos resultados con gonadotrofinas heterólogas aun en peces filogenéticamente cercanos (Zohar 1988, Estay y col 1996, Peter y Yu 1997, Patiño 1997).

\section{GONADOTROFINAS PURIFICADAS}

Numerosas investigaciones fueron realizadas con el fin obtener una gonadotrofina purificada cuya actividad pueda estandarizarse y proveerse en forma regular, de manera de reemplazar los extractos crudos de hipófisis (Donaldson y col 1972, Harvey y Hoar 1980, Davy y Chouinard 1980). Uno de los productos más utilizados por algún tiempo fue la gonadotrofina parcialmente purificada de salmón (SG-G100), pero las altas dosis requeridas (aprox. $10 \mathrm{mg} / \mathrm{kg}$ ) incrementaban los costos; el desarrollo de respuesta inmune en el pez receptor, su sensibilidad térmica y su bajo efecto producto de su especificidad redujeron sus aplicaciones productivas (Harvey y Hoar 1980, Mylonas y Zohar 2001 , Zohar y Mylonas 2001).

\section{GONADOTROFINA CORIONICA HUMANA (hCG)}

Con el fin de optimizar los resultados obtenidos en la sincronización de la maduración de peces, a principio de la década del 70 se llegó a investigar con gonadotrofinas de mamíferos, especialmente gonadotrofina coriónica de yegua y humana (hCG), purificada desde la orina de mujeres embarazadas (Harvey y Hoar 1980, Katzman y Doisy 1932 citado por Zohar y Mylonas 2001). Consecuentemente, la hCG ha sido utilizada en la inducción del desove de algunas especies de peces cultivadas hoy en día (Harvey y Hoar 1980, Donaldson y Hunter 1983, Billard y Breton 1985, Rowland 1988, Cerqueira y col 1997) y su éxito por lo general se ha atribuido a una actividad semejante a la LH (Hoar 1969, Chaudhuri 1976 citado por Harvey y Hoar 1980).

Durante su aplicación, la hCG se utiliza en una dosis simple, lo que reduce la manipulación de los peces en comparación al uso de la técnica de hipofisación. La efectividad de una dosis única se debe probablemente al largo tiempo en circulación que permanece la hormona (Ohta y Tanaka 1997). Las concentraciones utilizadas se encuentran entre 100 y 4.000 UI por kg de peso del pez. Los resultados de su uso se mejoran notoriamente cuando se utiliza en combinación con la inyección de extractos de hipófisis (Harvey y Hoar 1980, Amutio y col 1986). La ventaja de esta hormona es que ella actúa directamente sobre la gónada y no requiere la activación de la glándula hipófisis, actuando así mucho más rápido, induciendo la maduración final del oocito, la espermiación y la puesta. Sin embargo, la hCG puede causar inmunorreacciones en el pez receptor, reduciendo o eliminando el efecto de la hormona en inyecciones posteriores (Patiño 1997).

Hormonas liberadoras de gonadotrofinas (GnRH y LH-RH) y sus análogos (GnRHa y LH-RHa). Los métodos más modernos de inducción a la ovulación se han centrado en la aplicación de hormonas liberadoras de gonadotrofinas (GnRH). Estas son moléculas pequeñas (decapéptidos) que realizan el control de la glándula hipófisis en su producción de gonadotrofinas (LH y FSH o GtH-I y GtH-II) (Montero y Dufour 1996, Patiño 1997). Con la creación de análogos sintéticos (LH-RHa y GnRHa) que resultaron más potentes y de mayor duración que los nativos, se generó su masificación (Zohar 1988, Peter y Yu 1997, Patiño 1997). Los preparados sintéticos o análogos son nonapéptidos (Estay y col 1996, Donaldson 1997). En la mayoría de ellos se han sustituido los aminoácidos de las posiciones 6, 7 u 8 en relación a la molécula de GnRH de mamíferos y carecen de glicina en posición 10 (Estay y col 1996); siendo utilizados en numerosas especies (Billard y col 1983, Carrillo y Zanuy 1995) como la carpa (Davy y Chouinard 1980, Peter y col 1988); salmónidos (Billard y Breton 1985, Estay 1996, Kitahashi y col 1998a); lubina (Sorbera y col 1996); loja (Peter y col 1988); en la lisa (Mugil cephalus) (Lee y Tamaru 1988); en "milkfish" 
(Chanos chanos) (Tamaru y col 1988 y Marte y col 1988); en "rabbitfish" (Siganus guttatus) (García y col 1993); en perca amarilla (Perca flavescens) (Dabrowski y col 1994); esturión del Atlántico (Acipenser oxyrinchus) (Moler y Fletcher 1999), entre otras especies.

Las ventajas de la aplicación de hormonas liberadoras $(\mathrm{GnRH})$ en la inducción de la puesta en peces sobre los preparados de GtH son resumidas por Zohar y Mylonas (2001):

- Las GnRH son pequeñas moléculas que no generan respuestas inmunes en los peces receptores, por lo que pueden ser utilizadas repetidas veces.

- Las GnRH "reparan” las alteraciones endocrinas producidas por el cautiverio y llevan a los peces a su maduración sexual plena.

- Las GnRH se producen a nivel del hipotálamo, el cual controla la secreción de otras hormonas importantes en la homeostasis del pez, como son la prolactina, somatotropina y tirotropina.

- El poder sintetizarlas y obtenerlas en forma pura evita el riesgo de transmitir enfermedades a los peces receptores.

- Finalmente, la estructura molecular de estas hormonas es muy similar en muchos peces, lo que permite su uso en un gran número de especies con gran efectividad.

Una inyección de GnRH induce un incremento inmediato en los niveles plasmáticos de $\mathrm{GtH}$ en muchos peces, pero durante un periodo corto de tiempo durante el cual se debe activar la maduración final de los oocitos, la espermiación o la puesta. La corta vida media de las GnRH es producto de la acción de endopeptidasas ubicadas en la hipófisis, hígado y riñón (Peter y Yu 1997) que actúan en los enlaces peptídicos en posición 5-6 y 9-10, transformándola en pequeños fragmentos inactivos. En los análogos sintéticos, se han sustituido en esas posiciones aminoácidos $\mathrm{D}$ y con etilamida, respectivamente, esto los hace resistentes a la acción enzimática y presentan una vida media mucho mayor que las hormonas nativas (aproximadamente 23 y $5 \mathrm{~min}$, respectivamente) y la calidad de las puestas no difiere de la obtenida en los peces controles (Carrillo y Zanuy 1995, Peter y Yu 1997; Zohar y Mylonas 2001, Mylonas y Zohar 2001 ${ }^{\mathrm{b}}$ ).

De esta manera, la GnRHa permanece en circulación mucho más tiempo y estimula una mayor producción de GtH por más tiempo. Además, pueden presentar una mayor afinidad por los receptores de la hipófisis (Zohar y Mylonas 2001, Mylonas y Zohar 2001 b). Estos factores dan como resultado una acción 30 a 100 veces más potente en los análogos, lo que ha hecho que estas hormonas sustituyan prácticamente todas las otras sustancias utilizadas en las terapias hormonales aplicadas a peces. Se debe considerar además que los análogos requieren dosis mucho más bajas que las hormonas nativas. De 1 a $15 \mathrm{mg}$ de $\mathrm{GtH} / \mathrm{kg}$ de pez frente a 1 a $100 \mu \mathrm{g}$ de GnRHa/kg de pez.
La dopamina es una hormona que actúa a nivel de la hipófisis en algunos peces inhibiendo la liberación de $\mathrm{GtH}$ y atenuando la acción de la GnRH en las gonadotrofinas (Zohar 1988, Peter y Yu 1997). Por lo anterior, antagonistas de la acción de la dopamina (domperidone, pimozide, reserpine o metoclopramida) han sido utilizadas en combinación con GnRHa en la inducción de la maduración en algunas especies (Peter y col 1988, Peter y Yu 1997, Patiño 1997, Zohar y Mylonas 2001, Mylonas y Zohar 2001 b), estando bien establecida su efectividad en ciprínidos y algunas especies de agua dulce (Zohar y col 1995). En cambio, la utilización de inhibidores dopaminérgicos tiene resultados pobres y es poco recomendable su uso en la lubina americana y otras especies marinas (Carrillo y col 1995). Thomas y col (1995) señalan que el sistema dopaminérgico inhibidor de la secreción de GtHII está bloqueado en especies como la corvina, "spotted sea trout", "red drum" y "Atlantic croaker".

La aromatasa es una enzima que realiza la conversión de testosterona (T) a $17 \beta$-estradiol (E2) en el ovario; esta hormona es la encargada de estimular al hígado para la producción de vitelogenina. Recientes investigaciones han evaluado el uso de inhibidores de aromatasa (fadrozole) junto a GnRHa con el fin de inducir la maduración final del oocito y la espermiación en peces. Un inhibidor de la aromatasa bloquea la conversión de T a E2 en el ovario y de esta manera se genera el incremento en los niveles del esteroide inductor de la maduración $(17 \alpha, 20 \beta$ -dihidroxi-4-pregne-3-one) y de la ovulación (Alfonso y col 1999 citado por Zohar y Mylonas 2001).

\section{METODOS DE APLICACION}

La forma de aplicación de estos productos ha sido estudiada por numerosos autores y evaluadas en muchas especies (Peter y col 1988, Zohar 1988, Donaldson 1997, Patiño 1997). La mayoría de ellas se inician con aplicaciones en un medio líquido para llegar en la actualidad al uso de compuestos biodegradables capaces de regular la liberación de la hormona incorporada en el producto. A continuación se realiza una descripción breve de las formas de aplicación más utilizadas en sistemas de producción en acuicultura.

\section{MEDIO LIQUIDO}

Suero fisiológico. Su uso ha sido aplicado desde la incorporación de la hipofisación, sin embargo, la mayor desventaja se traduce en que toda la hormona incorporada en él queda inmediatamente a disposición del torrente circulatorio del pez, de manera que su entrega es durante un breve periodo. Por lo anterior, será necesaria más de una inyección para inducir la ovulación en la mayoría de las especies. 
Implantes. Los primeros sistemas de liberación lenta fueron preparados a base de colesterol (Crim y col 1988, Sherwood y col 1988, García 1989, 1993) y posteriormente evaluados en varias especies de salmónidos (Donaldson y Hunter 1983, Weil y Crim 1983 citado por Zohar y Mylonas 2001, Crim y col 1988, Bromage y Cumaranatunga 1988, Billard 1992, Estay y col 1996, Zohar y Mylonas 2001), en Lates calcarifer (García, 1989), en Dicentrarchus labrax (Carrillo y col 1995, Forniés y col 2001), en Paralichthys orbignyanus (Cerqueira y col 1997) y en numerosas especies de peces de interés comercial. En la actualidad se pueden preparar mezclados con celulosa y sus proporciones determinan implantes de liberación lenta (durante ocho semanas) o rápida (durante ocho días). Regularmente cada "pellet" contiene entre 25 y $250 \mu \mathrm{g}$ de hormona y pueden ser aplicados en forma intramuscular o intraperitoneal (Crim y col 1988, Sherwood y col 1988, Varas 1996, Zohar y Mylonas 2001, Mylonas y Zohar 2001 b). Estos "pellet" entregan cerca del $50 \%$ de la hormona contenida dentro de 2-3 hrs y el $90 \%$ a las 5-6 hrs (Crim y col 1988). Sin embargo, Sherwood y col 1988 reportan que un implante preparado con $95 \%$ de colesterol y $5 \%$ de celulosa sólo libera entre un 36 a $38 \%$ de la hormona contenida después de veintiocho días. Este mayor tiempo de liberación facilita la acción biológica de la hormona y además amplía el tiempo que tiene el piscicultor para implantar los peces, los que no necesariamente deben ser inyectados cuando la vesícula germinal se encuentra migrando, como ocurre cuando se utilizan medios líquidos.

Microesferas. La alta rigidez, baja degradabilidad y acelerada entrega de la hormona en los implantes han llevado a buscar nuevos compuestos que faciliten su manejo y prolonguen el tiempo de liberación de la hormona contenida. Debido a lo anterior, en peces se ha realizado la evaluación de polímeros biodegradables, de menor tamaño y mayor flexibilidad. Las evaluaciones más exitosas se han realizado con polímeros de Ac. Láctico y Ac. Glicólico (LGA), Ac. Sebásico (Fad-sa) y Acetato de etilen vinílico (EVAc). Sus mayores ventajas se centran en el prolongado tiempo de liberación de la hormona, su carácter biodegradable, facilidad de aplicación y fabricación (Zohar y Mylonas 2001, Mylonas y Zohar $2001^{\mathrm{b}}$ ). Forniés y col (2001) comparan la efectividad de diferentes sistemas de liberación lenta en D. labrax, determinando que las microesferas indujeron un mayor número de puestas y sin efectos negativos en la calidad de los gametos producidos en comparación a implantes de EVAc con $50 \mu \mathrm{g} / \mathrm{kg}$ o una inyección en suero salino con $15 \mu \mathrm{g} / \mathrm{kg}$.

Además de estas formas variadas de inducción a la maduración final del oocito en peces de cultivo y que requieren de repetidos manejos que pueden llevar a la muerte del animal (Patiño 1997), también se ha examinado en peces el uso potencial de la administración oral de las hormonas. Aunque los resultados han sido promisorios, este método no ha sido estudiado completamente (Thomas y Boyd 1989, Solar y col 1990, McLean y col 1991, Sukumasavin y col 1992, Patiño 1997, Donaldson 1997). Thomas y Boyd (1989) informan de la utilización de GnRHa administrada en el alimento para inducir la puesta sin deteriorar la calidad de gametos en la trucha de mar (Cynoscion negulosus), carpa (C. carpio), trucha arcoiris $(O$. mykiss) y en el pez gato africano (Clarias gariepinus). Esta técnica de suministro de la hormona presenta perspectivas promisorias para la acuicultura, ya que reduce el manejo de los peces a una simple biopsia ovárica para elegir el momento apropiado en que se debe iniciar el tratamiento (Patiño 1997).

\section{CUIDADOS PARA SU APLICACION}

Cualquiera sea el método de aplicación o el vehículo utilizado en la inducción a la puesta que se pretenda realizar, es fundamental conocer el estado de maduración en el que se encuentran las células germinales. Recordando siempre que los tratamientos sólo serán efectivos si los oocitos se encuentran en su fase de maduración final y el reproductor es manejado adecuadamente, manteniéndolo con bajos niveles de estrés. Importantes investigadores del tema recomiendan la aplicación de los tratamientos durante las primeras horas de la mañana y además identificar el momento en que la vesícula germinal se encuentra migrando a la periferia del oocito, momento en que la efectividad de una inyección será más efectiva ${ }^{1}$.

\section{CONCLUSIONES}

En la actualidad existe una variada gama de terapias hormonales disponibles para el piscicultor y que son factibles de utilizar en sus procesos productivos. ¿Cuál es la más recomendable?, dependerá de la especie que se cultiva y de los recursos con que se cuenta para adquirirla. La tendencia es a masificar el uso de productos análogos por su alta eficiencia, mayor vida útil y la ausencia de respuesta inmune en los peces receptores. Se debe continuar buscando reducir los costos de estos productos y buscar formas de aplicación menos invasivas para el pez (y menos agotadoras para el personal de la piscicultura). En la actualidad en la salmonicultura chilena, por ejemplo, el uso de implantes de GnRHa es una actividad rutinaria a pesar de su alto costo.

\section{RESUMEN}

En la presente revisión bibliográfica se analiza el estado actual del control artificial de la madurez sexual en peces de cultivo utilizando terapias hormonales, analizando en forma crítica las ventajas y desventajas de las diferentes estrategias tecnológicas existentes para un cultivador. Se discute desde los primeros intentos exitosos realizados por Houssay (1930) en Argentina, hasta las últimas y cada vez más exitosas técnicas de liberación lenta y el uso cada vez mayor de inhibidores de la dopamina. Además, se discute el uso de metodologías no traumáticas que a la fecha no han resultado del todo exitosas.

\footnotetext{
R. Billard, comunicación personal.
} 


\section{AGRADECIMIENTOS}

Se agradece el apoyo del proyecto FONDEF D06I1020 y a la Dirección de Investigación de la Universidad Católica de Temuco a través del proyecto DGIUCTemuco N²007-DGI-CDA-05. Además, al Dr. Adrián Hernández por la elaboración del resumen en inglés.

\section{REFERENCIAS}

Abellan E, B Basurco. 1999. Marine finfish species diversification; current situation and prospects in mediterranean aquaculture. Options méditerranéennes. 24, 1-139.

Amutio VG, A Espinach-Ros, A Fortuny. 1986. Field-induced breeding of the dorado, Salminus macillosus Valenciennes. Aquaculture 59, $15-21$.

Berlinsky D, W King, R Hodson, C Sullivan. 1997. Hormone induced spawning of summer flounder Paralichthys dentatus. $J$ World Aquacult Soc 28, 79-86.

Billard R. 1986. Spermatogenesis and spermatology of some teleost fish species. Reprod Nutr Dev 26, 877-920.

Billard R. 1989. Endocrinology and fish culture. Fish Physiol Biochem 7, 49-58.

Billard R. 1992. Reproduction in rainbow trout: sex differentiation, dynamics of gametogenesis, biology and preservation of gametes. Aquaculture 100, 263-298.

Billard R, B Breton. 1985. Control of reproduction and fish farming. In: Lofts B, Holmes WN (eds). Comparative endocrinology. Hong Kong, Hong Kong University Press, Pp 1221-1229.

Billard R, C Weil, G Barnabe. 1983. Induction de l'ovulation et stimulation de la spermiation par le LHRH ou un analogue de LHRH associe ou non au pimozide chez quelques espèces de poissons teleosteens. Bases Biologiques de l'Aquaculture. Montpellier. IFREMER. Actes de Colloques $\mathrm{N}^{\circ} 1$, Pp 321-332.

Bromage N. 1995. Broodstock Management and Seed Quality - General Considerations. In: Bromage N, Roberts R (eds). Broodstock management and egg and larval quality. Blackwell Science, Oxford, UK, Pp 1-24.

Bromage N, R Cumaranatunga. 1988. Egg production in the rainbow trout. In: Muir JF Roberts RJ (eds). Recent advances in aquaculture. Blackwell Scientific Publications, Oxford, UK, Pp 64-138.

Bromage N, M Porter, C Randall. 2001. The environmental regulation of maturation in farmed finfish with special reference to the role of photoperiod and melatonin. Aquaculture 197, 63-98.

Brown JA, LW Crim. 1998. Progress on the development of yellowtail flounder (Pleuronectes ferrugineus) for aquaculture. Proceedings of the Special Session on Marine Fish Culture Aquaculture Canada '97. Bull Aquacult Assoc Can (98-1), Pp 16-17.

Campbell PM, TG Pottinger, JP Sumpter. 1992. Stress reduces the quality of gametes produced by rainbow trout. Biol Reprod 47, 1140-1150.

Carrillo M, S Zanuy. 1995. Manipulación de la reproducción de los teleósteos y calidad de las puestas. En: Castelló F, Calderer A (eds). Actas del $V^{\circ}$ Congreso Nacional de Acuicultura. Sant Carles de la Rápita, España, Pp 1-9.

Carrillo M, S Zanuy, F Prat, J Cerda, J Ramos, E Mañanos, N Bromage. 1995. Sea bass (Dicentrarchus labrax). In: Bromage N, Roberts FJ (eds). Broodstock management and egg and larval quality. Blackwell Science Ltd, Cambridge, UK, Pp 138-168.

Cerqueira VR, R Mioso, JAG Macchiavello, AM Brugger. 1997. Ensaios de inducao do linguado (Paralichthys orbignyanus Valenciennes, 1839). B Inst Pesca, 24 ( ${ }^{\circ}$ especial), 247-254.

Courtois F, F Takashima, R Billard. 1986. Stimulation of spermiation following repeated injection of carp pituitary homogenates in the carp. B Jpn Soc Sci Fish 52, 995-997.

Crim LW, NM Sherwood, CE Wilson. 1988. Sustained hormone release. II. Effectiveness of LHRH analog (LHRHa) administration by either single time injection or cholesterol pellet implantation on plasma gonadotropin levels in a bioassay model fish, the juvenile rainbow trout. Aquaculture 74, 87-95.

Dabrowski K, A Ciereszko, L Ramseyer, D Culver, P Kestemont. 1994. Effects of hormonal treatment on induced spermiation and ovulation in the yellow perch (Perca flavescens). Aquaculture 120, 171-180.

Davy FB, A Chouinard 1980. Induced fish breeding in Southeast Asia. Report of a workshop held in Singapore, IDRC-178e, Pp 48.

Donaldson EM. 1997. The role of biotechnology in sustainable aquaculture. In: Bardach JE (ed). Sustainable aquaculture. John Wiley and Sons Inc., New York, USA, Pp 101-126.

Donaldson EM, F Yamazaki, H Dye, WW Philleo. 1972. Preparation of gonadotropin from salmon (Oncorhynchus tshawytscha) pituitary glands. Gen Comp Endocrinol (E.U.) 18, 469-481.

Donaldson EM, GA Hunter. 1983. Induced final maduration, ovulation and spermiation in cultured fishes. In: Hoar WS, Randall DJ, Donaldson EM (eds). Fish Physiology. Reproduction. Vol. 9B. Academic Press, Orlando, USA, Pp 351-403.

Donaldson EM, RH Devlin, F Piferrer, I Solar. 1996. Hormones and sex control in fish with particular emphasis on salmon. Asian Fisheries Science 9, 1-8.

Edwards DJ. 1978. Salmon and trout farming in Norway. Fishing News Books Limited, Farnham, Surrey, England.

Estay F. 1988. Análisis de algunos factores determinantes de alteraciones en la maduración gonadal y fertilidad de trucha arco iris (Salmo gairdnerii) cultivada. Tesis de Master, Universidad de Chile, Santiago, Chile.

Estay F, H Cerisola, V Téllez. 1994. Biología del desarrollo y reproducción artificial en la trucha arcoiris. CONICYT-FONDEF, Serie Publicaciones para la acuicultura $\mathrm{N}^{\circ} 1$.

Estay F, N Díaz, R Neira, X García. 1995ª . Reproductive performance of cultured female coho salmon in Chile. The Progressive FishCulturist 59, 36-40.

Estay F, N Díaz, L Valladares, G Dazarola. $1995^{\text {b }}$. Manejo reproductivo de salmónidos. CONICYT-FONDEF. Serie Publicaciones para la acuicultura $\mathrm{N}^{\circ} 2$.

Estay FJ, AM Ronco, L.G. Cáceres. 1996. Respuesta ovulatoria y niveles séricos de GtH II alcanzados en hembras de trucha arco iris (Oncorhynchus mykiss) inducidas a ovular con GnRHa D-Ala6. Arch Med Vet 38, 73-78.

Fontaine M. 1975. Physiological mechanism in the migration of marine and amphihaline fish. Adv Mar Biol 13, 241-355.

Foo JR, J Lam. 1993. Retardation of ovarian growth and depression of serum steroid levels in the tilapia, Oreochromis mossambicus, by cortisol implantation. Aquaculture 115, 133-143.

Forniés MA, E Mañanós, M Carrillo, A Rocha, S Laureau, CC Mylonas, Y Zohar, S Zanuy. 2001. Spawning induction of individual European sea bass females (Dicentrarchus labrax) using different GnRHadelivery systems. Aquaculture 202, 221-234.

García L. 1989. Dose-dependent spawning response of mature female sea bass, Lates calcarifer (Bloch), to pelleted luteinizing hormonereleasing hormone analogue (LHRHa). Aquaculture 77, 85-96.

García L. 1993. Sustained production of milt in rabbitfish, Siganus guttatus Bloch, by weekly injection of luteinizing hormone-releasing hormone analogue (LHRHa). Aquaculture 113, 261-267.

Gordon MR, KC Klotins, VM Campbell, MN Cooper. 1987. Farmed salmon broodstock management. Ministry of Environment Victoria, B.C. Industrial Research Assistance Program National Research Council of Canada, Sea-1 Aquafarms Ltd, Vancouver, BC, Canada.

Harvey BJ, WS Hoar. 1980. Teoría y práctica de la reproducción inducida en los peces. Centro Internacional para el Desarrollo, Ottawa, Canadá.

Hoar W. 1969. Reproduction. In: Hoar WS, Randall DJ (eds). Fish Physiology. Vol. III. Academic Press Inc, USA, Pp 1-59.

Kitahashi T, D Alok, H Ando, M Kaeriyama, Y Zohar, H Ueda, A Urano. $1998^{\mathrm{a}}$. GnRH analog stimulates gonadotropin II gene expression in maturing sockeye salmon. Zool Sci 15, 761-765. 
Kitahashi T, A Sato, D Alok, M Kaeriyama, Y Zohar, K Yamauchi, A Urano, H Ueda. 1998 b. Gonadotropin-releasing hormone analog and sex steroids shorten homing duration of sockeye salmon in lake Shikotsu. Zool Sci 15, 767-771.

Kjorsvik E, A Mangor-Jensen, I Holmefjord. 1990. Egg quality in fishes. Adv Mar Biol 26, 71-113.

Larsson DGJ, CC Mylonas, Y Zohar, LW Crim. 1997. Gonadotropin releasing hormone-analogue (GnRH-A) advances ovulation and improves the reproductive performance of a cold-water batch-spawning teleost the yellowtail flounder (Pleuronectes ferrugineus). Can J Aquat Fish Sci 54, 1957-1964.

Lee CS, CS Tamaru. 1988. Advances and future prospects of controlled maturation and spawning of grey mullet (Mugil cephalus L.) in captivity. Aquaculture 74, 63-73.

Marte CL, LW Crim, NM Sherwood. 1988. Induced gonadal maturation and rematuration in milkfish: Limited success with chronic administration of testosterone and gonadotropin-releasing hormone analogues (GnRH-A). Aquaculture 74, 131-145.

McLean E, DB Parker, CM Warby, NM Sherwood, EM Donaldson. 1991. Gonadotropin release following oral delivery of luteinizing hormonereleasing hormone and its superactive analogue (des-Gly ${ }^{10}\left[\mathrm{D}-\mathrm{Ala}^{6}\right]$ LHRH ethylamide) to 17ß-oestradil-primed coho salmon, Oncorhynchus kisutch (Walbaum). J Fish Biol 38, 851-858.

Miura T, T Kasugai, Y Nagahama, K Yamauchi. 1995. Acquisition of potential for sperm motility in vitro in Japanese eel Anguilla japonica. Fisheries Sci 61, 533-534.

Mohler JW, JW Fletcher. 1999. Induced spermiation in wild Atlantic stugeons held captive up to six years. $N$ Am J Aquacult 61, 70-73.

Montero M, S Dufour. 1996. Gonadotropin-releasing hormones (GnRH) in fishes: evolutionary data on their structure, localization, regulation and function. Zoological Studies 35, 149-160.

Mylonas CC, LC Woods, P Thomas, RW Schulz, Y Zohar. 1998 a Hormone profiles of captive striped bass (Morone saxatilis) during spermiation, and long-term enhancement of milt production. $J$ World Aquacult Soc 29, 379-392.

Mylonas CC, LC Woods, P Thomas, Y Zohar. 1998 . Endocrine profiles of female striped bass (Morone saxatilis) during post-vitellogenesis, and induction of final oocyte maturation via controlled-release GnRHadelivery systems. Gen Comp Endocrinol 110, 276-289.

Mylonas CC, Y Zohar. 2001 ${ }^{\mathrm{a}}$. Endocrine regulation and artificial induction of oocyte maturation and spermiation in basses of the genus Morone. Aquaculture 202, 205-220.

Mylonas CC, Y Zohar. 2001 ${ }^{\mathrm{b}}$. Use of GnRHa-delivery systems for the control of reproduction in fish. Fish Biology and Fisheries 10, 463-491.

Nagahama Y. 1983. The functional morphology of teleost gonads. In Hoar WS, Randall DJ (eds). Fish Physiology. Vol. IX A. Academic Press Inc, New York, USA, Pp 223-268.

Ohta, H, H Tanaka, 1997. Relationship between serum levels of human chorionic gonadotropin (hCG) and 11-ketotestosterone after a single injection of hCG and induced maturity in the male Japanese eel, Anguilla japonica. Aquaculture 153, 123-134.

Pagán-Font FA, J Zimet. 1985. Propagación artificial de las carpas chinas. Programa de Cooperación Técnica de la Organización de las Naciones Unidas para la Agricultura y la Alimentación (FAO), Pp 37.

Pankhurst NW, G Van Der Kraak. 1997. Effects of stress on reproduction and growth of fish. In: Iwama GK, Pickering AD, Sumpter JP, Schreck CB (eds). Fish stress and health in aquaculture. Cambridge University Press, UK, Pp 73-93.

Patiño R. 1997. Manipulations of the reproductive system of fishes by means of exogenous chemicals. The Progressive Fish-Culturist 59, 118-128.

Patiño R, G Yoshizaki, T Peter, H Kagawa. 2001. Gonadotropic control of ovarian follicle maturation: the two-stage concept and its mechanisms. Comparative Biochemistry and Physiology, Part B 129, 427-439.

Peter RE, HR Lin, G Van der Kraak. 1988. Induced ovulation and spawning of cultured freshwater fish in China: Advances in application of GnRH analogues and dopamine antagonists. Aquaculture 74, 1-10.
Peter RE, HR Lin, G Van der Kraak, M Little. 1993. Releasing hormones, dopamine antagonists and induced spawning. In: Muir JF, Roberts RJ (eds). Recent Advances in Aquaculture. Blackwell Scientific, Oxford, UK, Pp 25-30.

Peter RE, KL Yu. 1997. Neuroendocrine regulation of ovulation in fishes: basic and applied aspects. Rev Fish Biol Fisher 7, 173-197.

Prat F, S Zanuy, N Bromage, M Carrillo. 1999. Effects of constant short and long photoperiod regimes on the spawning performance and sex steroid levels of female and male sea bass. J Fish Biol 54, 125-137.

Redding JM, R Patiño. 1993. Reproductive physiology. In: Evans DH (ed). The physiology of fishes. CRC Press, USA, Pp 503-534.

Rodríguez L, I Begtashi, S Zanuy, M Carrillo. 2000. Development and validation of an enzyme immunoassay for testosterone: Effects of photoperiod on plasma testosterone levels and gonadal development in male sea bass (Dicentrarchus labrax, L.) at puberty. Fish Physiol Biochem 23, 141-150.

Rowland, SJ, 1988. Hormone-induced spawning of the Australian freshwater fish Murray cod, Maccullochella peeli (Mitchell)(Percichthyidae). Aquaculture 70, 371-389.

Schreck CB, W Contreras-Sanchez, MS Fitzpatrick. 2001. Effects of stress on fish reproduction, gamete quality, and progeny. Aquaculture 197, 3-24.

Sherwood NM, LW Crim, J Carolsfeld, SM Walters. 1988. Sustained hormone release. I. characteristics of in vitro release of gonadotropin-releasing hormone analogue (GnRH-A) from pellets. Aquaculture 74, 75-86.

Solar I. 2002. Biotecnología aplicada a la acuicultura. Aquanoticias Internacional $\mathrm{N}^{\circ}$ 66, Pp 6-10.

Solar I, E McLean, IJ Baker, NM Sherwood, EM Donaldson. 1990. Induced ovulation of sablefish (Anoplopoma fimbria) following oral administration of des gly super(10)-(D-Ala super(6) LH-RH ethylamide. Fish Physiol Biochem 8, 497-499.

Sorbera LA, CC Mylonas, S Zanuy, M Carrillo, Y Zohar. 1996. Sustained administration of GnRHa increases milt volume without altering sperm counts in the sea bass. J Exp Zool 276, 361-368.

Sower SA, CB Schreck. 1980. Sexual maturation of coho salmon (Oncorhynchus kisutch): accelerated ovulation and circulating steroid hormone and ion levels of salmon in freshwater and seawater. Proc. No. Pac. Aquaculture Symp, Anchorage, Alaska, Pp 227-235.

Sukumasavin N, W Leelapatra, E McLean, EM Donaldson. 1992. Orally induced spawning of thai carp (Puntius gonionotus, Bleeker) following co-administration of des Gly super(10)(D-Arg super(6)) sGnRH ethylamide and domperidone. J Fish Biol 40, 477-479.

Tamaru CS, CS Lee, CD Kelley, JE Banno, PY Ha, K Aioda, I Hanyu. 1988. Characterizing the stage of maturity most receptive to an acute LHRH-analogue therapy for inducing milkfish (Chanos chanos) to spawn. Aquaculture 74, 147-163.

Thomas P, CR Arnold. 1993. Environmental and hormonal control of reproduction in sciaenid fish. En: Muir JF, Roberts RJ (eds). Recent advances in aquaculture IV. Blackwell Scientific Publications, Cambridge UK, Pp 31-42.

Thomas P, NW Boyd. 1989. Dietary administration of an LHRH analogue induces spawning of spotted seatrout (Cynoscion nebulosus). Aquaculture 80, 363-370.

Thomas P, CR Arnold, GJ Holt. 1995. Red drum and other Sciaenids. In: Bromage N, Roberts FJ (eds). Broodstock management and egg and larval quality. Blackwell Science Ltd, Cambridge UK, Pp 118-137.

Thorgaard GH. 1995. Biotechnological approaches to broodstock management. In: Bromage NR, Roberts RJ (eds). Broodstock management and egg and larval quality. Blackwell Science, Oxford UK, Pp 76-93.

Tucker J. 1994. Spawning by captive serranid fishes: a review. $J$ World Aquacul Soc 25, 345-359.

Van Winkoop A, LPM Timmermans, HJ Goos. 1994. Stimulation of gonadal and germ cell development in larval and juvenile carp (Cyprinus carpio L.) by homologous pituitary extract. Fish Physiology and Biochemistry 13, 161-171.

Varas A. 1996. Uso de implantes hormonales comerciales para sincronizar el desove de Oncorhynchus kisutch (Walbaum, 1782). Tesis. Universidad Católica del Norte, Chile. 
Vermeirssen ELM, AP Scott, CC Mylonas, Y Zohar. 1998. Gonadotropinreleasing hormone agonist stimulates milt fluidity and plasma concentrations of 17,20 $\beta$-dihydroxylated and $5 \beta$-reduced, $3 \alpha$-hydroxylated C21 steroids in male plaice (Pleuronectes platessa). Gen Comp Endocrinol 112, 163-177.

Vermeirssen ELM, R Shields, C Mazorra de Quero, AP Scott. 2000. Gonadotrophin-releasing hormone agonist raises plasma concentrations of progestogens and enhances milt fluidity in male Atlantic halibut (Hippoglossus hippoglossus). Fish Physiol Biochem 22, 77-87.

Wagner E, R Arndt, B Hilton. 2002. Physiological stress responses, egg survival and sperm motility for rainbow trout broodstock anesthetized with clove oil, tricaine methanosulfonate or carbon dioxide. Aquaculture 211, 353-366.

Yambe H, M Shindo, F Yamazaki. 1999. A release pheromone that attracts males in the urine of mature female masu salmon. $J$ Fish Biol 55, 158-171.
Zanuy S, F Prat, M Carrillo, N Bromage. 1995. Effects of constant photoperiod on spawning and plasma 17ß-oestradios levels of sea bass (Dicentrarchus labrax). Aquat Living Resour 8, 147-152.

Zohar Y. 1988. Gonadotropin releasing hormone in spawning induction in teleosts: basic and applied considerations. Reproduction in fish: basic and applied aspects in endocrinology and genetics. Tel-Aviv, Israel, 10-12 November 1986. Ed. INRA, Paris 1988. Les Colloques de l'INRA 4, 47-60.

Zohar Y. 1996. New approaches for the manipulation of ovulation and spawning in farmed fish. Bull Natl Res Inst Aquacult, Suppl 2, 43-48.

Zohar Y, CC Mylonas. 2001. Endocrine manipulations of spawning in cultured fish: from hormones to genes. Aquaculture 197, 99-136. 
